\title{
La investigación sobre el marketing relacional: un análisis de contenido de la literatura 2007-2008*
}

\author{
Research on Relational Marketing: \\ an Analysis on Literature Content 2007-2008
}

\author{
José Luis Wakabayashi'
}

Doctoral Research Proposal

Recibido: 20 julio, 2010

Aceptado: 29 septiembre, 2010

\section{Resumen}

El propósito de este artículo es realizar una revisión de la literatura sobre el Marketing Relacional, elaborar un análisis de contenido de la misma para hacer una clasificación y, por último, proveer al teórico y al práctico de Marketing de una bibliografía por temas. Se realizó una búsqueda de literatura sobre Marketing Relacional en 55 revistas especializadas. Cada artículo fue tomado como unidad de análisis. La clasificación de todas las unidades en categorías excluyentes fue elaborada por dos investigadores independientes para asegurar la validez de la clasificación. El análisis de contenido arrojó cinco categorías excluyentes: objetivos, constructos, instrumentos, aplicaciones industriales y temas para los años 2007 y 2008. El estudio, además, ordena a los artículos según criterios de región, año e industria estudiada para esos mismos años. La clasificación por industrias implicó reordenar a los autores de los textos revisados según criterio universal de codificación de actividades económicas CIIU.

Palabras claves: Marketing Relacional, relaciones con el consumidor, administración de la información, revisión de literatura.

\begin{abstract}
The purpose of this article is to perform a literature review on Relational Marketing, to prepare a content analysis of it for classification and to provide to Marketing academics and practitioners with a bibliography by subjects. A search on Relational Marketing articles was performed in 55 specialized journals. Each article was taken as a unit of analysis. The classification of the units in excluding categories was prepared by two independent researchers to confirm the validity of the classification. The analysis of content considered five excluding categories: objectives, constructs, instruments, industrial applications and subjects for the years 2007 and 2008. The study, at the same time, classifies the articles according the criteria of region, region and studied industry for these years. The classification for industries implied reordering all the authors of the reviewed texts according to universal criteria of codification of economics activities CIIU.
\end{abstract}

Keywords: Relational Marketing, relationship with consumer, information management, literature review

* El autor desea agradecer especialmente la invalorable ayuda de María Elena Gushiken en el desarrollo de esta propuesta doctoral.

1. Profesor Auxiliar de la Universidad ESAN. 


\section{INTRODUCCIÓN}

Desde su inserción formal, en los inicios de la década pasada, el enfoque relacional del Marketing se ha asociado, según los autores consultados, a la construcción, el desarrollo y el mantenimiento de vínculos e intercambios beneficiosos entre la empresa y sus clientes. Sin embargo, existen un sinnúmero de definiciones para el Marketing Relacional. Para efectos de la investigación, se toman dos definiciones que engloban sus características generales.

Por un lado, para Jobber y Fahy (2006), el Marketing Relacional es el proceso de crear, desarrollar y mejorar las relaciones con los clientes y otros actores que participan de la relación, conocidos como stakeholders. Por otro lado, Boone y Kurtz (2007) afirman que el Marketing Relacional se refiere al desarrollo, el crecimiento y el mantenimiento de largo plazo del intercambio efectivo de relaciones con clientes, proveedores, empleados y otros socios para lograr beneficios mutuos. Ambas definiciones coinciden en que el propósito central del Marketing Relacional es crear valor mutuo a partir de un proceso de gestión en que se desarrollan las relaciones duraderas con los clientes; es decir, se aprovechan las sinergias que se pueden establecer en dichas relaciones, para mantener la rentabilidad a lo largo del ciclo de vida del cliente. También coinciden en que los clientes pueden ser todos aquellos actores involucrados en sus relaciones.

Este trabajo pretende tomar la perspectiva de la teoría del Marketing Relacional y enriquecerla mediante la revisión de la literatura publicada sobre este campo. Su objetivo es seguir el legado del autor Kallol Das (2009) y proveer una clasificación de la literatura así como una bibliografía global que pueda ser útil tanto para investigadores como para aquellos involucrados en la gestión. El texto presenta una sección con la metodología usada para la clasificación y el análisis de contenido. Una subsiguiente sección trata sobre los resultados seguida por las conclusiones y las implicaciones de la investigación. Al final se incluyen cuáles serían algunas futuras líneas de investigación.

\section{METODOLOGÍA}

En esta sección se presenta una revisión de la investigación sobre Marketing Relacional publicada en revistas especializadas entre los años 2007 y 2008. La literatura fue sometida a un análisis de contenido con el propósito de clasificación. Dicho análisis de contenido es una poderosa técnica de reducción de datos, y su mayor beneficio es que se trata de una técnica sistemática y replicable para comprimir una gran cantidad de palabras de textos. A partir de ello se obtiene una menor cantidad de categorías, basadas en reglas específicas de codificación (Krippendorff, 1980; Weber, 1990; Stemler, 2001). Este análisis no es invasivo y sirve para lidiar con grandes volúmenes de información.

El proceso para el análisis de contenido siguió los cinco criterios sugeridos por Kassarjian y Robertson (1991) y Kolbe y Burnett (1991): muestreo, objetividad, reliabilidad, sistematización y cuantificación. La muestra se restringe a la literatura sobre Marketing Relacional encontrada en 55 revistas especializadas. Ellas fueron las mismas que Kallol Das trabajó en su análisis de contenido sobre la investigación del Marketing Relacional para los años 1994-2006. Justamente se decidió tomar las mismas revistas como muestra para realizar una comparación entre los contenidos hallados en dicho periodo investigado por Das y el periodo actual.

Para la selección de artículos se usó el criterio de búsqueda Relationship Marketing ${ }^{1}$, tanto para el título como para las palabras clave y el resumen en los artículos comprendidos entre los años 2007 y 2008. Este es el mismo criterio que Das aplicó. Los artículos que respondieron a esta búsqueda fueron revisados para seleccionar solamente aquellos que fueran efectivamente afines al Marketing Relacional. Se obtuvieron 111 artículos relacionados a la materia de manera general, y que conforman la literatura referida al Marketing Relacional para las revistas seleccionadas en el periodo elegido. A partir de los artículos escogidos, se realizó un análisis de contenido de cada uno de ellos.

1 Todos los criterios de búsqueda se realizaron con el marcador universal de definiciones Tesauro. 
Los resultados pueden generalizarse a esta totalidad de la literatura encontrada (Malhotra, 2004; Das, 2009); sin embargo, no puede generalizarse al universo de la literatura del Marketing Relacional.

Para el presente estudio, la unidad de análisis es el texto completo del artículo (Stock, 1997). Cada artículo fue estudiado de manera independiente por los dos investigadores para identificar el lugar adecuado en la categorización. Luego de una discusión, ambos investigadores decidieron clasificar la totalidad de los artículos usando el modelo analítico que se detalla en la Figura 1. Este modelo es una versión expandida del modelo desarrollado por Lindgreen (2001) en el que se clasifican las diversas disciplinas del Marketing Relacional. Incidentalmente, es el mismo que K. Das aplicó para su clasificación y asegurar la objetividad de la categorización.

Como se muestra en la Figura 1, toda la literatura sobre Marketing Relacional puede ser clasificada en las siguientes cinco categorías: objetivos, definición de constructos, instrumentos, temas de discusión y aplicaciones en la industria. En cuanto a sus objetivos, el Marketing Relacional se ha definido en cuanto a satisfacción del cliente, portafolio de clientes, retención del cliente, lealtad, entre otros. La clasificación según constructos del Marketing Relacional se relaciona con términos como confianza, compromiso, cooperación, cercanía, calidad de la relación, entre otros. En cuanto a las herramientas, se considera al Marketing Relacional como marketing directo, marketing de base de datos, marketing de uno a uno, gestión de la relación con el cliente, marketing de la lealtad, sociedades, etc. Todas ellas representan herramientas/instrumentos para establecer relaciones con los clientes.

Por otro lado, algunos artículos también incorporan la privacidad, impacto cultural, impacto del género, impacto de la tecnología, impacto del marketing de sociedades/alianzas. Estas categorías fueron clasificadas como temas de discusión porque representan tópicos que influenciar al Marketing Relacional o, en su defecto, ser influenciados por él. Finalmente, se planteó la clasificación según su aplicación en industrias, lo que correspondería al empleo de programas de Marketing Relacional en organizaciones e industrias/sectores específicos.

Cada investigador realizó una clasificación de los artículos siguiendo estos cinco criterios por separado, con una afinidad del $90 \%$ en sus respectivas clasificaciones. Holsti (1969) sugiere un mínimo de 85\% de concordancia para que la categorización sea aceptable. Se toma este porcentaje entre investigadores como una manera de medir la confiabilidad del estudio, tal como proponen Kolbe y Burnett. El desacuerdo entre los investigadores fue resuelto con una discusión concienzuda basada en el modelo presentado en la Figura 1 y apoyada por la opinión de un tercer investigador independiente.

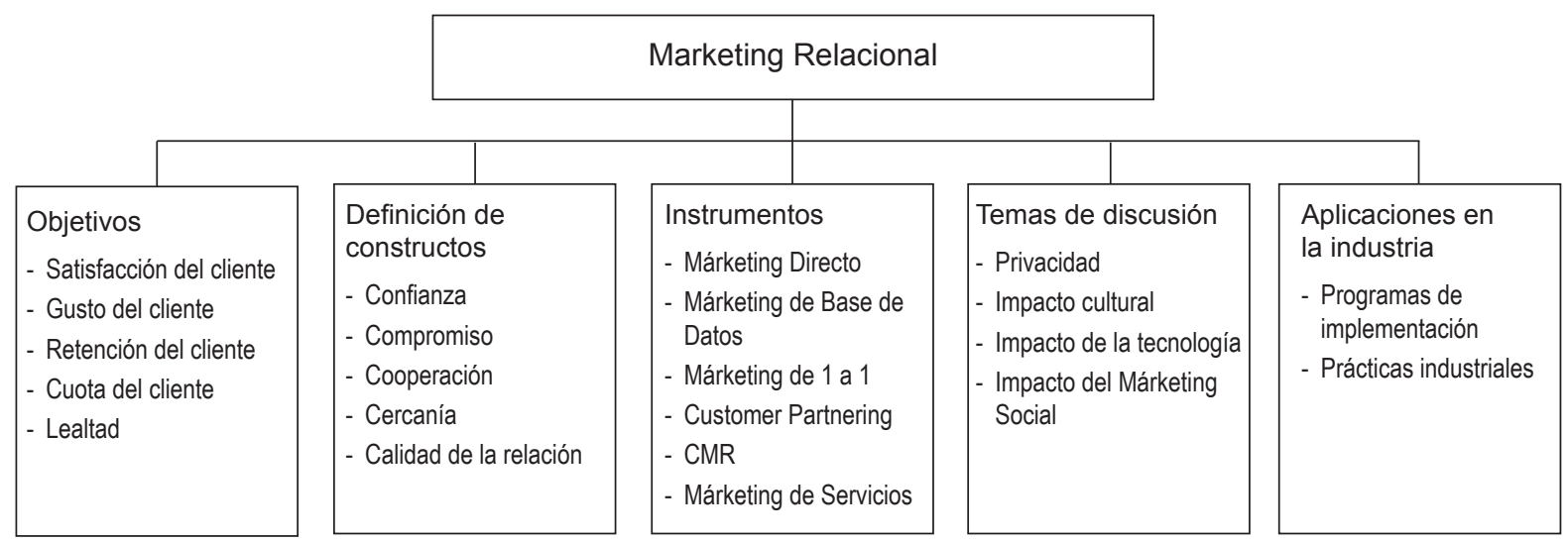

Figura 1. Modelo de categorías de clasificación para el Marketing Relacional

Fuente: Adaptado de Lindgreen (2001) y Das (2009). 
Se clasificaron los artículos nuevamente de acuerdo con las nuevas opiniones y finalmente se cuantificaron los resultados.

\section{RESULTADOS}

\section{Clasificación de la literatura revisada}

La Tabla 1 muestra una bibliografía global de los artículos académicos correspondientes a las cinco categorías ya mencionadas. Este es un recurso útil tanto para los profesionales de campo como para los investigadores que están buscando artículos de investigación del Marketing Relacional en algún área específica.

\section{Distribución de artículos según categoría}

La Tabla 2 muestra la distribución de los artículos con sus frecuencias según las categorías acordadas y según año de publicación.

Puede observarse que la mayoría de los artículos se encuentran clasificados dentro de la categoría Cons-

Tabla 1.

Bibliografía según categorías de contenido*

\begin{tabular}{cl}
\hline Categoría & \multicolumn{1}{c}{ Bibliografía } \\
\hline \multirow{2}{*}{ Objetivos } & $\begin{array}{l}\text { Cross, M. et al. (2007); Jaramillo, F. et al. (2007); Saren (2007); Jamal, A. \& Adelowore, A. (2008); } \\
\text { Mekonnen, A. et al. (2008); Dadzie, K. et al. (2008); Hobbes, R. \& Rowley, J. (2008); Doherty, B. (2008); } \\
\end{array}$ Nicholson, J. et al. (2008). \\
& Plewa, C. \& Quester, P. (2008); Cofta, P. (2007); Lee, T. \& Jun, J. (2007); Sinisalo, J. et al. (2007); Sichtmann, \\
Constructos & C. (2007); Murphy, P. (2007); Antón, C. (2007); Terawatanavong, C. (2007); Wang, S. (2007); García \\
& Rodríguez, N. (2007); Glynn, M. (2007); Fink, R. (2007); Palmer, R. (2007); García de Madariaga, J. \& Valor, \\
& C. (2007); Connell, J. \& Voola, R. (2007); Plewa, C. \& Quester, P. (2007); Eisingerich, A. \& Bell, S. (2007); \\
& Ward, T. \& Dagger, T. (2007); Wong, Y.H. (2007); Oly Ndubisi, N. (2007); Oly Ndubisi (2007b); Kottila, \\
& M. \& Rönni, P. (2008); Lu, H. (2008); Jarratt, D. (2008); Davis, P. (2008); Martenson, R. (2008); Heffernan, T. \\
& et al. (2008); Brodie, R. et al. (2008); Barry, J. \& Terry, T. (2008); Du Sig Choi, P. \& Palihawadana, D. (2008); \\
& Harmon, T. \& Griffiths, M. (2008); Sirgy, M. \& Lee, D. (2008); Barry, J. et al. (2008); Wong, Y.H. et al. (2008); \\
& Maritz, A. \& Nieman, G. (2008); Wang, S. \& Davis, L. (2008); Carter, T. (2008); Victoria Bordonaba-Juste, M. \\
& \& Polo-Redondo, Y. (2008); Athanasopoulou, P. (2008); Dimitriadis, S. \& Stevens, E. (2008); Chen, Z. X. et \\
& al. (2008); Hsieh, A.T. \& Li, CH. K. (2008); Endo, S. \& Kincade, D. (2008); Fawcett, S. et al. (2008); Soosay, \\
& C. (2008); Chiao, Y. et al. (2008); Liang, C. et al. (2008).
\end{tabular}

Herramientas Valsecchi, M. et al. (2007); Schierholz, R. et al. (2007); Trim, P. \& Lee, Y. I. (2008); Fung, P. (2007); Kingshott, R. \& Pecotich, A. (2007); Nguyen, T. et al. (2007); Hughes, T. et al. (2007); Tang, L. et al. (2007); Ballantyne, D. \& Aitken, R. (2007); Luo, X. \& Donthu, N. (2007); Marin, L. \& Ruiz, S. (2007); Knox, S. \& Gruar, C. (2007); Ferguson, R. \& Hlavinka, K. (2007); Wendlandt, M. \& Schrader, U. (2007); Zineldin, M. \& Philipson, S. (2007); Ferguson, R. \& Hlavinka, K. (2007b); Roemer, E. (2007); Ryals, L. \& Holt, S. (2007); Ryals, L. \& Rogers, B. (2007); Richard, J. et al. (2007); Rowley, J. (2007); Triki, A. et al. (2007); Nätti, S. \& Still, J. (2007); Metallo, G. et al. (2007); Clements, M. et al. (2008); Battor, M. (2008); EspinoRodríguez, T. \& Rodríguez-Díaz, M. (2008); Foo, M. et al. (2008); Harrigan, P. et al. (2008).

Temas de Maulana, A. \& Eckhardt, G. (2007); Hansen, S. (2008), Greenyer, A. (2008); Badrinarayanan, V. \& Arnett, discusión D. (2008); McKinley-Floyd, L. \& Shrestha, N. (2008); Zupancic, D. (2008); Lin, J.C. \& Chen, CH. (2008).

Aplicaciones en la industria

No se encontraron artículos que pudieran clasificarse dentro de esta categoría.

* La relación completa de la bibliografía, que incluye las revistas donde aparecen los artículos de los autores citados en esta tabla, puede encontrarse en el siguiente link: ABCD. 
Tabla 2.

Distribución de artículos según categoría

\begin{tabular}{lrrr}
\hline \multicolumn{1}{c}{ Categoría } & \multicolumn{1}{c}{2007} & \multicolumn{1}{c}{2008} & \multicolumn{1}{c}{ Total } \\
\hline Objetivos & $3(4.48 \%)$ & $7(15.91 \%)$ & $10(9.01 \%)$ \\
Constructos & $39(58.21 \%)$ & $26(59.09 \%)$ & $65(58.56 \%)$ \\
Herramientas & $24(35.82 \%)$ & $5(11.36 \%)$ & $29(26.13 \%)$ \\
Temas de discusión & $1(1.49 \%)$ & $6(13.64 \%)$ & $7(6.31 \%)$ \\
Aplicación en industrias & $0(0 \%)$ & $0(0 \%)$ & $0(0 \%)$ \\
Total & $67(100 \%)$ & $44(100 \%)$ & $111(100 \%)$ \\
\hline
\end{tabular}

tructos $(58.56 \%)$, con una estabilidad con respecto del total para ambos años en la producción de artículos en esta categoría. En su investigación, Das halló un declive constante en la investigación del Marketing Relacional con respecto de sus constructos. Sin embargo, al parecer esta tendencia no está sucediendo en los años examinados en el presente estudio. En cuanto a la categoría Objetivos, la proporción de artículos ha crecido considerablemente de un año al siguiente, alcanzando un $9.01 \%$ del total de artículos. Este porcentaje se mantiene con lo hallado por Das $(9.09 \%)$. Se observa que los artículos referidos a las herramientas han ido decreciendo en el tiempo (de 24 a 5 artículos), aunque en total representa una proporción mayor a la del período $1994-2006$ (de $20.10 \%$ a $26.13 \%$ ).

En cuanto a los temas de discusión que influyen sobre el Marketing Relacional, los artículos publicados han aumentado considerablemente de un año al siguiente y mantienen la ascendencia que Das anotara anteriormente. Con respecto de la aplicación de programas de Marketing Relacional en industrias específicas, no se encontró ningún artículo que pudiera identificarse con esta categoría, mientras que para años anteriores al 2006, los artículos sobre el particular representaban un importante $12.44 \%$. El hecho de que la mayoría de artículos se hayan clasificado dentro de la categoría Constructos implica que existe un esfuerzo por parte de los investigadores de crear teoría. Por su parte, la investigación del Marketing Relacional en cuanto a sus herramientas muestra el interés por encontrar aplicaciones prácticas de esta orientación dentro de la disciplina.

\section{Distribución de artículos según revistas}

La Tabla 3 muestra el número de artículos con los que cada revista analizada contribuyó en los años 2007 y 2008. Del total de 113 artículos publicados entre el 2007 y 2008, las revistas que han contribuido con más de diez artículos son European Journal of Marketing, con 24 artículos, y el Journal of Business \& Industrial Marketing, con 18. Le siguen International Journal of Bank Marketing (9), Marketing Intelligence \& Planning (8), Journal of Services Marketing (7), Journal of Strategic Marketing (7). Estas mismas revistas contribuyeron con la mayor cantidad de artículos para los años anteriores al 2007 (Das, 2009).

\section{Distribución de artículos según región}

La Tabla 4 muestra la distribución de los artículos encontrados según la región donde se elaboró el artículo (texto teórico), o la región donde se realizó el estudio (artículo investigativo).

Estados Unidos, Inglaterra y Australia son los países donde más investigación y publicaciones se han realizado, con 25, 24 y 12 artículos publicados, respectivamente; este resultado coincide con la tendencia hallada en los años anteriores al 2007. China (5), Taiwán (6), Nueva Zelandia (5) y Finlandia (5) también continúan como centros de investigación. Resulta interesante el aumento de publicaciones españolas (de $3.28 \%$ a $8.40 \%$ ). Por otro lado, el único país de la región latinoamericana fue Argentina (una publicación), que concuerda con la tendencia de años 
Tabla 3.

Distribución de artículos de investigación según revistas

\begin{tabular}{|c|c|c|c|}
\hline Nombre de la revista & 2007 & 2008 & Total \\
\hline Asia Pacific Journal of Marketing and Logistics & 1 & - & 1 \\
\hline Behaviour \& Information Technology & 1 & - & 1 \\
\hline British Food Journal & - & 3 & 3 \\
\hline British Journal of Management & - & - & - \\
\hline BT Technology Journal & - & - & - \\
\hline Business Process Management Journal & 4 & - & 4 \\
\hline Business Strategy Review & - & 1 & 1 \\
\hline Campus Wide Information Systems & - & - & - \\
\hline Construction Management and Economics & - & - & - \\
\hline Decision Sciences & - & - & - \\
\hline European Business Review & 2 & - & 2 \\
\hline European Journal of Marketing & 19 & 5 & 24 \\
\hline Expert Systems & - & - & - \\
\hline Handbook of Business Strategy & - & - & - \\
\hline IMA Journal of Management Mathematics & - & - & - \\
\hline Industrial Management \& Data Systems & - & 2 & 2 \\
\hline Information Management \& Computer Security & 1 & - & 1 \\
\hline Information Systems and e Business Management & - & - & - \\
\hline Information Technology and Management & - & - & - \\
\hline International Review of Retail, Distribution \& Consumer Research & - & - & - \\
\hline International Journal of Bank Marketing & 5 & 4 & 9 \\
\hline International Journal of Contemporary Hospitality Management & - & - & - \\
\hline International Journal of Educational Management & - & - & - \\
\hline International Journal of Retail \& Distribution Management & - & - & - \\
\hline International Journal of Service Industry Management & 1 & - & 1 \\
\hline International Marketing Review & 1 & - & 1 \\
\hline Journal of Business \& Industrial Marketing & 9 & 9 & 18 \\
\hline Journal of Business Ethics & 3 & 1 & 4 \\
\hline Journal of Change Management & - & - & - \\
\hline Journal of Consumer Marketing & 4 & 1 & 5 \\
\hline Journal of European Industrial Training & - & - & - \\
\hline Journal of Intellectual Capital & - & - & - \\
\hline Journal of Knowledge Management & 1 & - & 1 \\
\hline Journal of Management History & - & - & - \\
\hline Journal of Management Studies & - & - & - \\
\hline Journal of Market Focused Management & - & - & - \\
\hline
\end{tabular}




\begin{tabular}{|c|c|c|c|}
\hline Journal of Marketing Communications & - & - & - \\
\hline Journal of Marketing Practice: Applied Marketing Science & - & - & - \\
\hline Journal of Services Marketing & 3 & 4 & 7 \\
\hline Journal of Small Business Management & - & - & - \\
\hline Journal of Strategic Marketing & 4 & 3 & 7 \\
\hline Library Management & - & - & - \\
\hline Management Decision & - & - & - \\
\hline Managerial Auditing Journal & - & - & - \\
\hline Managing Leisure & - & - & - \\
\hline Managing Service Quality & - & 2 & 2 \\
\hline Marketing Intelligence \& Planning & 4 & 4 & 8 \\
\hline Marketing Letters & - & - & - \\
\hline Qualitative Market Research: An International Journal & 2 & 1 & 3 \\
\hline Supply Chain Management: An International Journal & - & 3 & 3 \\
\hline The Journal of Enterprise Information Management & 1 & - & 1 \\
\hline The Service Industries Journal & 1 & 2 & 3 \\
\hline The TQM Magazine & - & - & - \\
\hline Total Quality Management & 1 & - & 1 \\
\hline TQM \& Business Excellence & - & - & - \\
\hline Total de artículos por revista & 68 & 45 & 113 \\
\hline
\end{tabular}

anteriores. Es importante anotar que Argentina no es un país que sea representativo de la mayoría de los países de Latinoamérica, ya que sus ventas no están concentradas en el canal minorista.

\section{Distribución de artículos según industrias}

La Tabla 5 muestra la distribución de los artículos revisados según la industria a la que pertenecen las empresas investigadas en los artículos. Se debe resaltar que los artículos suman más de 111 debido a que muchas de las publicaciones son estudios que abarcan múltiples industrias. Incidentalmente, esta clasificación por industrias implicó el mayor reto para los investigadores.

En primer lugar, los autores de los artículos no aplicaron criterios conocidos de clasificación de las industrias que estudiaban en el marco de sus investigaciones; tampoco se encontró que se utilizara alguna clasificación internacional que permitiese realizar agru- paciones objetivas entre artículos. Ante ello, se estableció para cada uno de los artículos sectores económicos de acuerdo con el CIIU (Clasificación Industrial Internacional Uniforme), como una manera de estandarizar los textos, que finalmente son la unidad de análisis de la investigación. El CIIU clasifica todas las actividades económicas establecida por la ONU con el fin de ofrecer un conjunto de categorías de actividades económicas que permita reunir y difundir datos estadísticos de acuerdo con esas actividades (ONU 2009).

Aún así, en algunos casos los autores de los artículos tampoco llegaron a definir con claridad el tipo de actividad estudiada, lo que hacía mucho más difícil la clasificación. Este fue el caso de la industria «reparación técnica», que podría haber hecho referencia a reparaciones técnicas de muy variados tipos: mecánica, de computadoras, etc. Por otro lado, una reparación técnica puede ser ofrecida por un profesional individual, por una empresa, o ser parte de un proceso mayor 
Tabla 4.

Distribución de artículos según región

\begin{tabular}{|c|c|c|c|c|}
\hline País o región & 2007 & 2008 & Total & Contribución \\
\hline Alemania & 2 & 1 & 3 & $2.29 \%$ \\
\hline Asia Pacífico & 1 & 0 & 1 & $0.76 \%$ \\
\hline Australia & 9 & 3 & 12 & $9.16 \%$ \\
\hline Argentina & 0 & 1 & 1 & $0.76 \%$ \\
\hline China & 1 & 4 & 5 & $3.82 \%$ \\
\hline Corea & 1 & 2 & 3 & $2.29 \%$ \\
\hline Costa de Marfil & 0 & 1 & 1 & $0.76 \%$ \\
\hline Dinamarca & 2 & 0 & 2 & $1.53 \%$ \\
\hline España & 8 & 3 & 11 & $8.40 \%$ \\
\hline Estados Unidos & 13 & 12 & 25 & $19.08 \%$ \\
\hline Europa & 2 & 0 & 2 & $1.53 \%$ \\
\hline Finlandia & 4 & 1 & 5 & $3.82 \%$ \\
\hline Francia & 1 & 2 & 3 & $2.29 \%$ \\
\hline Ghana & 0 & 1 & 1 & $0.76 \%$ \\
\hline Grecia & 0 & 2 & 2 & $1.53 \%$ \\
\hline Inglaterra & 16 & 8 & 24 & $18.32 \%$ \\
\hline Irlanda & 0 & 1 & 1 & $0.76 \%$ \\
\hline Italia & 2 & 1 & 3 & $2.29 \%$ \\
\hline Malasia & 2 & 0 & 2 & $1.53 \%$ \\
\hline Múltiple & 1 & 3 & 4 & $3.05 \%$ \\
\hline Nigeria & 0 & 1 & 1 & $0.76 \%$ \\
\hline Nueva Zelandia & 4 & 1 & 5 & $3.82 \%$ \\
\hline Rusia & 0 & 1 & 1 & $0.76 \%$ \\
\hline Suecia & 4 & 1 & 5 & $3.82 \%$ \\
\hline Suiza & 0 & 1 & 1 & $0.76 \%$ \\
\hline Taiwán & 2 & 4 & 6 & $4.58 \%$ \\
\hline Túnez & 1 & 0 & 1 & $0.76 \%$ \\
\hline Total & 76 & 55 & 131 & $100.00 \%$ \\
\hline
\end{tabular}

de servicio. Para estos casos, no pudo establecerse el CIIU, circunstancia que se consideró como parte de las limitaciones de la investigación.

Por otro lado, muchas investigaciones no consignaron en los artículos los sectores estudiados, limitándose a dejar entredicho que se estudiaban sectores que brindaban servicios o sectores que fabricaban o vendían bienes. En esos casos, se clasificó como «bienes», «servicios»o «bienes y servicios».

Continuando con la tendencia para los años anteriores al 2007, la industria más estudiada es la de servicios financieros y seguros, con $20.38 \%$ del total; este porcentaje indica el claro sesgo de la investigación hacia el campo de los servicios. Le sigue el de comercio al 
Tabla 5.

Distribución de artículos según industrias

\begin{tabular}{|c|c|c|c|c|}
\hline Industria & 2007 & 2008 & Total & Contribución \\
\hline Actividades artísticas, de entretenimiento y recreativas & 5 & 0 & 5 & $3.18 \%$ \\
\hline Actividades de alojamiento y de servicio de comidas & 0 & 5 & 5 & $3.18 \%$ \\
\hline Actividades de atención de la salud humana y de asistencia social & 1 & 2 & 3 & $1.91 \%$ \\
\hline Actividades de servicios administrativos y de apoyo & 6 & 2 & 8 & $5.10 \%$ \\
\hline Actividades de servicios de información & 1 & 0 & 1 & $0.64 \%$ \\
\hline Actividades financieras y de seguros & 20 & 12 & 32 & $20.38 \%$ \\
\hline Actividades inmobiliarias & 0 & 1 & 1 & $0.64 \%$ \\
\hline Actividades profesionales, científicas y técnicas & 4 & 3 & 7 & $4.46 \%$ \\
\hline $\begin{array}{l}\text { Administración pública y defensa; planes de seguridad } \\
\text { social de afiliación obligatoria }\end{array}$ & 1 & 0 & 1 & $0.64 \%$ \\
\hline Agricultura, ganadería, caza y actividades de servicios conexas & 0 & 4 & 4 & $2.55 \%$ \\
\hline Bienes & 6 & 3 & 9 & $5.73 \%$ \\
\hline Bienes (B2B) & 1 & 0 & 1 & $0.64 \%$ \\
\hline Bienes y servicios & 13 & 13 & 26 & $16.56 \%$ \\
\hline Bienes y servicios (B2B) & 1 & 0 & 1 & $0.64 \%$ \\
\hline $\begin{array}{l}\text { Comercio al por mayor, excepto el de vehículos automotores } \\
\text { y motocicletas }\end{array}$ & 16 & 0 & 16 & $10.19 \%$ \\
\hline $\begin{array}{l}\text { Comercio al por menor, excepto el de vehículos automotores } \\
\text { y motocicletas }\end{array}$ & 0 & 5 & 5 & $3.18 \%$ \\
\hline Construcción & 1 & 1 & 2 & $1.27 \%$ \\
\hline Electrónica & 0 & 1 & 1 & $0.64 \%$ \\
\hline Enseñanza & 0 & 1 & 1 & $0.64 \%$ \\
\hline Explotación de minas y canteras & 1 & 0 & 1 & $0.64 \%$ \\
\hline Industrias manufactureras & 6 & 3 & 9 & $5.73 \%$ \\
\hline Información y comunicaciones & 2 & 0 & 2 & $1.27 \%$ \\
\hline Mantenimiento técnico & 0 & 1 & 1 & $0.64 \%$ \\
\hline Organizaciones sin fines de lucro & 2 & 1 & 3 & $1.91 \%$ \\
\hline Otras actividades de servicios & 1 & 1 & 2 & $1.27 \%$ \\
\hline Servicios & 2 & 3 & 5 & $3.18 \%$ \\
\hline Servicios (B2B) & 1 & 0 & 1 & $0.64 \%$ \\
\hline Suministro de electricidad, gas, vapor y aire acondicionado & 2 & 0 & 2 & $1.27 \%$ \\
\hline Transporte y almacenamiento & 1 & 0 & 1 & $0.64 \%$ \\
\hline Utilidades & 1 & 0 & 1 & $0.64 \%$ \\
\hline Total & 95 & 62 & 157 & $100.00 \%$ \\
\hline
\end{tabular}


Tabla 6.

Distribución de artículos según tipo de investigación

\begin{tabular}{lrrrr}
\hline \multicolumn{1}{c}{ Tipo de investigación } & 2007 & 2008 & Total & Contribución \\
\hline Investigación & 57 & 37 & 94 & $83.19 \%$ \\
Revisión o propuesta teórica & 10 & 9 & 19 & $16.81 \%$ \\
Total & 67 & 46 & 113 & $100.00 \%$ \\
\hline
\end{tabular}

por mayor, con $10.19 \%$, las industrias manufactureras, con $5.73 \%$. Después se encuentran el comercio al por menor, las actividades recreativas, de servicio de comidas, salud humana y servicios administrativos, con $3.18 \%$ en general. Es significativo el decrecimiento de la investigación en torno al comercio al por menor: de $10.4 \%$ en años anteriores al 2007 a $3.18 \%$ para los años 2007 y 2008.

\section{Distribución de artículos según tipo de investigación}

La Tabla 6 muestra la distribución de artículos según el tipo de investigación realizada. Como puede observarse, el $83 \%$ de la contribución es de publicaciones que implican una investigación empírica, mientras que el $16.81 \%$ representa publicaciones de tipo teórico. Para el periodo 2002-2006, Das halló que el 35.88\% de los artículos eran teóricos o conceptuales. Es importante, entonces, resaltar el aumento de contribuciones desde la perspectiva de la investigación.

\section{CONCLUSIONES E IMPLICACIONES DE LA INVESTIGACIÓN}

Este estudio halló 111 artículos referidos al Marketing Relacional para las revistas seleccionadas. Se encontró que la mayoría de los artículos tratan el tema desde el punto de vista de sus constructos y herramientas, lo que implica que existe un esfuerzo por parte de los investigadores de crear teoría, así como de probar aplicaciones prácticas de esta orientación del Marketing.

Se pudo comprobar que los autores de los artículos publicados no establecen con claridad las actividades económicas que estudian, que se refleja en la poca meticulosidad de la sección metodológica de los artículos, los resúmenes, o en las palabras clave. Del mismo modo, ningún autor utiliza un criterio internacional de clasificación para detallar el sector económico estudiado. En ese sentido, se sugiere que las revistas exijan a los investigadores claridad en sus criterios de clasificación de las industrias estudiadas, que permitan además la búsqueda efectiva de los temas de investigación en las bases de datos, con mayor razón en estos últimos años en que el porcentaje de artículos de tipo investigativo está en aumento.

Por último, se pudo comprobar que el sector servicios sigue siendo el campo más estudiado por los investigadores del Marketing Relacional, cuya mayor incidencia se da en el área de servicios financieros y de seguros. Por otro lado, se comprobó que la mayoría de estudios están centrados en los Estados Unidos, Inglaterra y Australia, y que no existen estudios para la región latinoamericana, con excepción de un único estudio realizado en Argentina. 


\section{Referencias}

Boone L. E., \& Kurtz D. L. (2007). Contemporary Marketing. Harcourt College Publishers: Philadelphia.

Das, K. (2009). Relationship Marketing Research (19942006). An academic literature review and classification. Marketing Intelligence \& Planning, 27, 3.

Holsti, O. R. (1969). Content Analysis for the Social Sciences and Humanities, Addison-Wesley, Reader: Massachusetts.

Jobber, D., \& Fahy, J. (2006). Foundations of Marketing, The McGraw-Hill companies: Nueva York.

Kassarjian, H. H., \& Robertson, T. S. (1991), Consumer Research, Vol. 4, Prentice-Hall, Englewood Cliffs, Nueva Jersey.

Kolbe, R. H., \& Burnett, M. S. (1991). Content-analysis research: an examination of applications with directives for improving research reliability and objectivity. Journal of Consumer Research, 18, 243-250.

Krippendorff, K. (1980). Content Analysis: An Introduction to Its Methodology. Newbury Park, CA: Sage.
Lindgreen, A. (2001), “A framework for studying relationship marketing dyads", Qualitative Market Research: An International Journal, Vol. 4 No. 2, pp. $75-87,88$.

Malhotra, N. K. (2004), Marketing Research: An Applied Orientation, 4th ed., Pearson Education: Nueva Delhi.

ONU (2009). Clasificación Industrial Internacional Uniforme de todas las actividades económicas (CIIU). Departamento de Asuntos Económicos y Sociales. División de Estadística. Informes estadísticos. Serie M, No. 4/Rev. 4. Nueva York.

Stock, J. R. (1997), Applying theories from other disciplines to logistic. International Journal of Physical Distribution \& Logistics Management, 27(9/10), 515-39.

Stemler, S. (2001). An overview of content analysis. Practical Assessment, Research and Evaluation, 7, 17.

Weber, R. P. (1990). Basic Content Analysis (2nd ed.). Newbury Park, CA: Sage. 


\section{ANEXO}

Relación de revistas y journals que incluyen artículos tomados en cuenta para el análisis de contenido publicados entre los años 2007 y 2008 :

Asia Pacific Journal of Marketing and Logistics

Behaviour \& Information Technology

British Food Journal

Business Process Management Journal

Business Strategy Review

European Business Review

European Journal of Marketing

Industrial Management \& Data Systems

Information Management \& Computer Security

International Journal of Bank Marketing

International Journal of Service Industry Management

International Marketing Review

Journal of Business \& Industrial Marketing
Journal of Business Ethics

Journal of Consumer Marketing

Journal of Knowledge Management

Journal of Services Marketing

Journal of Strategic Marketing

Managing Service Quality

Marketing Intelligence \& Planning

Qualitative Market Research: An International Journal

Supply Chain Management: An International Journal

The Journal of Enterprise Information Management

The Service Industries Journal

Total Quality Management 